\title{
Optimization, Characteristics, and Functions of Alkaline Phosphatase From Escherichia coli
}

OPEN ACCESS

Edited by:

Nelson da Cruz Soares,

University of Sharjah, United Arab

Emirates

Reviewed by:

Jai K. Kaushik,

National Dairy Research Institute

(ICAR), India

Xia Xiong,

Institute of Subtropical Agriculture, Chinese Academy of Sciences (CAS),

China

Sumera Zaib,

University of Central Punjab, Pakistan

*Correspondence:

Qiang Tu

qiang.tu@siat.ac.cn

Jia Yin

jiayin@hunnu.edu.cn

†These authors have contributed equally to this work

Specialty section:

This article was submitted to Microbial Physiology and Metabolism, a section of the journa

Frontiers in Microbiology

Received: 19 August 2021 Accepted: 21 December 2021

Published: 21 February 2022

Citation:

Dong Y, Xia Y, Yin J, Zhou D, Sang Y, Yan S, Liu Q, Li Y, Wang L, Zhao Y, Chen C, Huang Q, Wang Y, Abbasi MN, Yang $H$, Wang $C$, Li J, Tu $Q$ and Yin J (2022) Optimization,

Characteristics, and Functions of Alkaline Phosphatase From Escherichia coli.

Front. Microbiol. 12:761189. doi: 10.3389/fmicb.2021.761189

\begin{abstract}
Yachao Dong ${ }^{1,2 t}$, Yandong Xia ${ }^{3 t}$, Jie Yin ${ }^{1}$, Diao Zhou', Yidan Sang ${ }^{1}$, Sufeng Yan', Qingshu Liu', Yaqi Li', Leli Wang', Ying Zhao', Cang Chen', Qiuyun Huang', Ying Wang ${ }^{1}$, Muhammad Nazeer Abbasi ${ }^{2}$, Huansheng Yang ${ }^{1}$, Chuni Wang ${ }^{1}$, Jianzhong $L^{1}{ }^{1}$, Qiang Tu ${ }^{2 *}$ and Jia Yin ${ }^{1 *}$
\end{abstract}

\footnotetext{
${ }^{1}$ Hunan Provincial Key Laboratory of Animal Intestinal Function and Regulation, Hunan International Joint Laboratory of Animal Intestinal Ecology and Health, The National and Local Joint Engineering Laboratory of Animal Peptide Drug Development, College of Life Sciences, Hunan Normal University, Changsha, China, ${ }^{2}$ CAS Key Laboratory of Quantitative Engineering Biology, Shenzhen Institute of Synthetic Biology, Shenzhen Institutes of Advanced Technology, Chinese Academy of Sciences (CAS), Shenzhen, China, ${ }^{3}$ College of Life Sciences and Technology, Central South University of Forestry and Technology, Changsha, China, ${ }^{4}$ Hunan Institute of Microbiology, Changsha, China
}

Weaning of piglets could increase the risk of infecting with Gram-negative pathogens, which can further bring about a wide array of virulence factors including the endotoxin lipopolysaccharide (LPS). It is in common practice that the use of antibiotics has been restricted in animal husbandry. Alkaline phosphatase (AKP) plays an important role in the detoxification and anti-inflammatory effects of LPS. This study investigated the protective effects of AKP on intestinal epithelial cells during inflammation. Site-directed mutagenesis was performed to modulate the AKP activity. The enzyme activity tests showed that the activity of the DelSig $D_{153} G-D_{330} N$ mutants in $B$. subtilis was nearly 1,600 times higher than that of the wild-type AKP. In this study, an in vitro LPS-induced inflammation model using IPEC-J2 cells was established. The mRNA expression of interleukin-(IL-) 6, IL-8, and tumor necrosis factor- $\alpha$ (TNF- $\alpha$ ) were extremely significantly downregulated, and that of ASC amino acid transporter 2 (ASCT-2), zonula occludens protein-1 (ZO-1), and occludin-3 (CLDN-3) were significantly upregulated by the DelSig $\mathrm{D}_{153} \mathrm{G}-\mathrm{D}_{330} \mathrm{~N}$ mutant compared with LPS treatment. This concludes the antiinflammatory role of AKP on epithelial membrane, and we are hopeful that this research could achieve a sustainable development for the pig industry.

Keywords: alkaline phosphatase, inflammatory factor, IPEC-J2 cell line, immunity, disease

\section{INTRODUCTION}

Weaning is a critical stage of mammalian postpartum growth and intestinal development. The early stages of weaning are usually associated with decreased performance (Pié et al., 2004: 6417) and increased incidence of intestinal diseases and diarrhea in animals (Fairbrother et al., 2005: 17-39), which would subsequently result in increasing the risk of infecting with Gram-negative pathogens, such as E. coli, S. typhimurium, etc. Therefore, it is necessary to prevent gut disorder and diseases by maintaining proper barrier function. Previously, farmers were gravitated to add different antibiotics into the feeds to prevent or reduce pathogenic infections. However, unregulated use of antibiotics has led to several problems, such as antibiotic resistance, adverse drug reactions, 
and accumulation of drug residues in animal products. Considering the mentioned issues, it is compelling to develop effective alternatives to replace antibiotics in animal feeds and to promote high-quality development of animal husbandry. Under existing commercial conditions, antibiotic alternatives include probiotics, organic acids, auxins, prebiotics, synbiotics, enzymes, antimicrobial peptides, hyperimmune eggs antibodies, clay, and metal (Gadde et al., 2017: 26-45).

The Gram-negative pathogens produce virulence factors including the endotoxin lipopolysaccharide (LPS). Functional LPS binds to Toll-like receptors (TLR-4) on immune cells through the main regulatory nuclear factor- $\kappa \mathrm{B}(\mathrm{NF}-\kappa \mathrm{B})$ pathway, consequently activating the secretion of proinflammatory cytokines (Goldberg et al., 2008: 3551-6), such as tumor necrosis factor- $\alpha$ (TNF- $\alpha)$, interleukin- (IL-)1, IL-6, IL-8, and IL-12 (O'Neill et al., 2013: 453-60). The increase in proinflammatory factors leads to the destruction and localization of tight junction protein levels, thereby increasing the passage of intestinal contents into the systemic circulation (Liu et al., 2016: 100917). The main functions of AKP include improvement of the intestinal barrier function (Liu et al., 2016: 1009-17), prevention of bacterial translocation (Estaki et al., 2014: 156506 ), and detoxification and anti-inflammatory effects of local or systemic intestinal LPS (Bilski et al., 2017: 9074601). AKP dephosphorylates the lipid A portion of LPS and no longer causes TLR-4 stimulation. AKP may even act as TLR-4 receptor antagonist in preventing its binding to the receptor (Molnár et al., 2012: 3254-9), which is essential for maintaining the normal intestinal homeostasis and inhibiting its transport across the intestinal mucosal barrier (Molnár et al., 2012: 3254-9).

Tight junctions are a kind of junction polyprotein complexes, which are located at the tip of the outer membrane of epithelial cells and serve as a barrier preventing solute diffusion across the intercellular space. The breakdown of tight junctions increases paracellular permeability, which allows the migration of harmful substances, such as pathogens and endotoxins, leading to tissue damage and inflammation. Previous studies have shown that the upregulation of zonula occludens protein1 (ZO-1) and occludins (CLDN) can inhibit the increase in intestinal permeability caused by tight junction disruption in weaned piglets. Therefore, maintaining appropriate expression levels of CLDN and ZO-1 are widely considered to be an effective target for the treatment of intestinal diseases (Omonijo et al., 2019: 615-24).

Previous studies have concluded that AKP is effective in reducing the risk of Gram-negative bacterial infection, blocking the secretion of LPS-activated proinflammatory cytokines. In addition, AKP is a regulator of intestinal mucosal permeability and appears to work through improving tight junction protein levels and localization (Liu et al., 2016: 1009-17). The cells were incubated with LPS detoxification by AKP, and mRNA transcriptional levels of inflammatory factors were monitored; the mRNA levels of ASC amino acid transporter 2 (ASCT-2), CLDN-3, and ZO-1 also were tracked. The proinflammatory cytokines, such as IL-6, IL-8, and TNF- $\alpha$, were related to the occurrence of immune function or pathological damage and significantly decreased, indicating that AKP can effectively alleviate the pathological injury of immune function to achieve anti-inflammation (Omonijo et al., 2019: 615-24). CLDN-3 and ZO-1 gene expression play an important role in maintaining barrier function and are exceptionally upregulated, revealing that AKP can effectively improve intestinal barrier function, possibly by preserving tight junction protein formation and integrity (Liu et al., 2016: 1009-17). ASCT-2 gene expression mainly absorbs neutral amino acids, such as glutamine, alanine, serine, and cysteine, and is greatly upregulated, providing important nutrients for cell metabolism (Xu et al., 2020: 111376).

E. coli has the ability to grow rapidly on cheap substrates, but it produces virulence factors, including the endotoxin LPS, and therefore cannot be used as feed additives. In recent years, the use of Gram-positive B. subtilis strains to produce recombinant proteins has been widely a concern by the academia and industry (Schallmey et al., 2004: 1; Liu et al., 2013: 6113-27). Previous studies have shown that $B$. subtilis can easily absorb extracellular genetic material as nutrients or expand the diversity of genotypes (Chen et al., 2005: 1456-60). In addition, B. subtilis has superior ability to exocrine proteins and other metabolites and without producing endotoxins, which makes it an ideal expression host for industrial and pharmaceutical applications (Fu et al., 2008: 287-92; Demain and Vaishnav, 2009: 297-306; Caspers et al., 2010: 1877-85; Cai et al., 2017: 70).

In this study, we have used homologous recombination coupled with site-directed mutagenesis techniques to construct a stable plasmid with high expression activity of the AKP in B. subtilis. By improving the activity of AKP to effectively prevent and control livestock and poultry diseases, reducing dependence on antibiotics, and promoting the healthy and harmonious development of the livestock and poultry industry, these meaningful findings of AKP would provide a certain reference for sustainable development of the pig industry.

\section{MATERIALS AND METHODS}

\section{Cell Line and Culture Conditions}

The IPEC-J2 cell line is derived from porcine IPEC intestinal epithelial cells and was purchased from Otwo Biotech ${ }^{1}$. The cells were grown and stored in a complete medium containing DMEM (89\% high sugar, Hyclone) supplemented with 10\% special-grade fetal bovine serum (phage-free low endotoxin, TIANHANG ${ }^{2}$ ) and $1 \%$ antibiotics (penicillin and streptomycin, Gibco). The AKP recombinant strain was cultured overnight at $37^{\circ} \mathrm{C}$ aerobically, and they were passed at least twice before the experiment. The culture supernatant was prepared by passing the supernatant through a sterile filter with a $0.22-\mu \mathrm{m}$ pore size (Hippo's). After filtering with a sterile syringe filter, the protein concentration was measured accurately through the Take $3^{\mathrm{TM}}$ multivolume plate (Thermo Scientific). The protein sample was normalized to $2 \mu \mathrm{g} / \mathrm{ml}$ and pretreated with $10 \mu \mathrm{g} / \mathrm{ml}$ of LPS at $37^{\circ} \mathrm{C}$ for $1 \mathrm{~h}$, then added into cells and incubated for $24 \mathrm{~h}$.

\footnotetext{
${ }^{1}$ http://www.otwobiotech.com/

${ }^{2}$ http://www.hzsjq.com/
} 


\section{Strains and Plasmids}

The strains used in this study are listed in Supplementary Table 1. GB05-dir was used for DNA recombineering (Fu et al., 2012: 440-6). It was incubated in LB aerobic medium (10 $\mathrm{g}$ of tryptone, $5 \mathrm{~g}$ of yeast extract, $5 \mathrm{~g}$ of $\mathrm{NaCl}$ per liter, and the solid medium is added with $1.4 \%$ agar) in a Thermo Mixer (Eppendorf) at $37^{\circ} \mathrm{C}, 900 \mathrm{rpm}$. Wild-type B. subtilis was used as host for heterologous expression of recombinant AKP. They were inoculated with GM I and then transferred into GM II medium for the plasmid transformation. The specific ingredients are as shown in Supplementary Table 2. The plasmid pSC101-BAD-ETgA-tet has the pSC101 origin, Larabinose inducible promoter, and tetracycline resistance gene. The plasmid pP43NMK-APN-Cm-Km contains a broad-host pP43NMK origin and conveys kanamycin and chloramphenicol resistance. Appropriate antibiotics were added at the following concentrations when necessary: kanamycin and chloramphenicol were used for E. coli strains at $10 \mu \mathrm{g} / \mathrm{ml}$, tetracycline was used for E. coli strains at $5 \mu \mathrm{g} / \mathrm{ml}$, and the concentration of L-arabinose used for induction was $1.4 \mathrm{mg} / \mathrm{ml}$.

\section{Screening of Alkaline Phosphatase Gene}

We used the NCBI online tool ${ }^{3}$ to search the open reading frame (initiation codon-termination codon) of AKP genes from different species. The signal peptide cleavage site of the obtained AKP was analyzed by online analysis software ${ }^{4}$. The porcine AKP genes have introns and are available through artificial gene synthesis [Bioengineering (Shanghai) Co., Ltd. ${ }^{5}$ ]. E. coli and B. subtilis AKP gene are obtained through PCR. The open reading frames of four AKP genes, including phoA (GenBank accession No. CP037857.2), phoE (GenBank accession No. CP053102.1), ncdF (GenBank accession No. CP052842.1), and apn are collected (GenBank accession No. AH012163.2). These expression plasmids containing AKP genes from different species were constructed by the Red/ET DNA recombineering technology. The AKP genes were placed behind the tetracycline inducible promoters $\left(\mathrm{P}_{\text {tet }}\right)$, and kanamycin and chloramphenicol resistance genes were also added into the constructs. The culture was induced with $0.2 \mu \mathrm{g} / \mu \mathrm{l}$ of tetracycline and incubated at $37^{\circ} \mathrm{C}$ for $4 \mathrm{~h}$. The AKP activity was detected with the AKP kit (Nanjing Jiancheng Institute of Biological Engineering ${ }^{6}$ ).

\section{Construction of Escherichia coli-Bacillus subtilis Shuttle Vector (pP43NMK)}

The plasmid pP43NMK-APN-Cm-Km was digested with PstI to expose the homology arms and to recover the 6,845-bp fragment as a linear vector. The AKP gene $p h o A$, phoE, and $n u d F$ were amplified from the plasmids pGB-Cm-ccdA-PtetphoA, pGB-Cm-ccdA-Ptet-phoE, and pGB-Cm-ccdA-Ptet-nudF. The oligonucleotides P43-phoA-5/P43-phoA-3, P43-phoE-5/P43phoE-3, and P43-nudF-5/P43-nudF-3 were used for PCR. The oligonucleotide sequences are listed in Supplementary Table 3.

\footnotetext{
${ }^{3}$ https://www.ncbi.nlm.nih.gov/

${ }^{4}$ www.cbs.dtu.dk/services/SignalP/

${ }^{5}$ https://mail.sangon.com/

${ }^{6}$ http://www.njjcbio.com/
}

Two hundred nanograms of PCR products and $200 \mathrm{ng}$ of pP43NMK linear vector were co-electroporated into the Larabinose-induced GB05-dir, and the recombinants were selected by kanamycin on LB agar plates at $37^{\circ} \mathrm{C}$ overnight and identified by restriction analysis and sequencing.

Alkaline phosphatase expression plasmids are modified and optimized to achieve high levels of production. First, the signal peptide of AKP gene was deleted, and DelSigphoA was prepared from the plasmid pGB-Cm-ccdA-Ptet-phoA by designing the fragment P43-DelSig-5/P43-phoA-3. Based on the characteristics of bioinformatics AKP, directed mutagenicity was carried out on the active sites of AKP based on the removal of signal peptides. Previous studies found that AKP mutant strains $\mathrm{D}_{101} \mathrm{~A}$ (Yun et al., 2013: 533-8), $D_{153} \mathrm{G}$ (Dealwis et al., 1995: 865-71), $\mathrm{E}_{322} \mathrm{Y}$ (Hou and Cui, 2012: 229-46), $\mathrm{K}_{328} \mathrm{Y}$ (Wojciechowski et al., 2002: 903-11), and $\mathrm{D}_{330} \mathrm{~N}$ (Le Du et al., 2002: 941-53) could improve AKP activity. A series of mutant expression plasmids were constructed to select mutant strains with high AKP activity. In this study, the toxin-antitoxin system (mazE-mazF system) was introduced into the expression plasmids to prevent loss of expression plasmids, the bacterial solution at a concentration of $10^{-5}$ was incubated for generations, and the stability of the plasmid was tested by counting the colony number.

\section{Simulation Protocol}

The initial structures of PhoA enzyme, DelSigphoA, and DelSigD ${ }_{153} \mathrm{G}-\mathrm{D}_{330} \mathrm{~N}$ were generated by I-tasser (Yang and Zhang, 2015: W174-81). Disodium phenyl phosphate was docked to these three proteins by Autodock (Morris et al., 2009: 2785-91), respectively. Molecular dynamic simulation was used to inspect the stability of each complex. All the calculations were performed by Amber 20 (Case et al., 2015: 1-884). Hydrogen atoms of protein were added by the Tleap module based on the Amber ff14SB force filed (Ponder and Case, 2003: 27-85). The systems were soaked in TIP3P (Jorgensen et al., 1983: 926-35) water box with a 10$\AA$ buffer. The sodium ions were added to neutralize the whole systems. The structures were then minimized by 4,000 steps of the steepest descent followed by 1,000 steps of conjugate-gradient descent. There was 80 ps of molecular dynamics simulations in the NPT ensemble during which the temperature was increased from 0 to $300 \mathrm{~K}$. After this initial relaxation, each system was simulated for $20 \mathrm{ps}$ in the NPT ensemble. All the systems were then simulated in the NVT ensemble in 100 ns with a time step of $2 \mathrm{fs}$. The trajectories obtained from the NVT ensemble were used for subsequent data analysis. For all three complexes, the root-mean-square-deviations (RMSDs) of all heavy atoms were calculated with the reference to the initial conformation.

\section{Precipitation of Extracellular Proteins Including Alkaline Phosphatase}

The strain of the DleSigphoA and DleSigD ${ }_{153} \mathrm{G}_{-} \mathrm{D}_{330} \mathrm{~N}$ were inoculated on LB agar plates containing kanamycin and cultured overnight at $37^{\circ} \mathrm{C}$. The AKP in the supernatant was precipitated by acid, acetone, ethanol, and salt. (1) Acid, $2 \% \mathrm{HCl}$, was slowly added into the supernatant so that the final $\mathrm{pH}$ of the mixed solution was reached to $4,4.5,5,5.5$, and 6 for $30 \mathrm{~min}$. 
(2) Acetone, the supernatant, was precipitated with $50 \%$ final concentration of acetone solution for $30 \mathrm{~min}$. (3) Ethanol, the supernatant, was precipitated with $50 \%$ final concentration of ethanol solution for $30 \mathrm{~min}$. (4) Salt, the supernatant, was precipitated with $50 \% \mathrm{NaCl}$ saturation solution for $30 \mathrm{~min}$. They were centrifuged at $4^{\circ} \mathrm{C}, 9,000 \mathrm{rpm} / \mathrm{min}$ for $30 \mathrm{~min}$ to collect precipitates and then suspended in $100 \mu \mathrm{l}$ of LB liquid medium to detect the AKP activity.

\section{Quantitative Real-Time PCR}

Total RNA was isolated from the cells by following the instructions of the manufacturer. The RNA concentration and $\mathrm{OD}_{260} / \mathrm{OD}_{280}$ value were accurately measured by using Take $3^{\mathrm{TM}}$ multivolume plate. Reverse transcription of $2 \mu \mathrm{g}$ of total RNA into cDNA used Prime Script ${ }^{\mathrm{TM}}$ RT reagent Kit with gDNA Eraser (Perfect Real Time) (RR047A, Takara) according to the instructions of the manufacturer. TB Green Premix Ex Taq ${ }^{\text {TM }}$ II (RR820A, Takara) was chosen for real-time PCR reaction to analyze the transcription levels of proinflammatory factors in Applied Biosystems Real Time PCR System (Thermo Fisher Scientific). The target genes were IL-6, IL-8, and TNF- $\alpha$, as well as ASCT 2, CLDN 3, and ZO-1. The reference genes $\beta$-actin and cyclophilin A (Cyc A) were being studied. The primers used for qPCR are listed in Supplementary Table 3. Two microliter of cDNAs were directly added in the PCR reaction mixture with a final volume of $20 \mu \mathrm{l}$, containing $10 \mu \mathrm{l}$ of TB Green Premix Ex Taq II (Tli RNaseH Plus) $(2 \times), 0.8 \mu \mathrm{l}$ of PCR Primer $(10 \mu \mathrm{M})$, and $0.4 \mu \mathrm{l}$ of ROX Reference Dye or Dye II $(50 \times)$. The amount of the reverse transcription reaction solution should not exceed $10 \%$ of the total volume of the PCR reaction solution. The thermal curve of all reactions was performed at $95^{\circ} \mathrm{C}$ for $3 \mathrm{~min}$, then $95^{\circ} \mathrm{C}$ for $5 \mathrm{~s}$ for 40 cycles, and at $60^{\circ} \mathrm{C}$ of $34 \mathrm{~s}$. The amplification curve and melting curve of real-time PCR were confirmed after the reaction was completed, and a standard curve for PCR quantification was prepared to ensure the specificity of the reaction. The amount of PCR product was calculated by Delta Delta $\mathrm{C}(\mathrm{T})$ method.

\section{RESULTS}

\section{Screening and Activity Optimization of the Alkaline Phosphatase}

Compared with the non-induced control, the AKP activity of the recombinants derived from $E$. coli was nearly increased by two times, while that from porcine and $B$. subtilis was not increased (Figure 1A). The results of enzyme activity assay showed that the activity derived from the pho $A$ gene of E. coli was still the highest in B. subtilis, which was 12.5 times higher than that in E. coli (Figure 1B). In order to further improve the enzymatic activity, the signal peptide was also deleted by Red/ET recombineering. The results revealed that the activity of DelSigphoA was significantly increased, and it was four times higher than that of the wild-type phoA (Figure 1C). A series of mutants were constructed using Red/ET recombinant engineering. Results revealed that the activity of the DelSigD ${ }_{153} \mathrm{G}-\mathrm{D}_{330} \mathrm{~N}$ mutants were the highest, which was 32 times higher than that of the DelSigphoA (Figure 1D).

The RMSDs of the generated structures of PhoA enzyme, DelSigphoA, and DelSigD ${ }_{153} \mathrm{G}-\mathrm{D}_{330} \mathrm{~N}$ were calculated. The RMSD between PhoA and DelSigphoA is $0.52 \AA, 0.60 \AA$ between PhoA and DelSigD ${ }_{153}$ G- $D_{330} \mathrm{~N}$, and $0.57 \AA$ between DelSigphoA and DelSigD ${ }_{153} G-D_{330} N$. The three complex structures of the docking results are shown in Supplementary Figures 1A-C and are the binding pockets of the PhoA-, DelSigphoA-, and DelSigD ${ }_{153} \mathrm{G}-\mathrm{D}_{330} \mathrm{~N}$-ligand complexes, respectively. It shows that the ligand strongly interacted with LYS-328 and ARG-166 of both DelSigphoA and DelSigD ${ }_{153} \mathrm{G}_{-} \mathrm{D}_{330} \mathrm{~N}$. Therefore, these LYS and $A R G$ may be the key residues for the enzymatic reaction. As can be seen, the side chain of ASP-153 in PhoA enzyme and DelSigphoA points to the center of the binding pocket. Mutation of this residue into GLY may eliminate steric hindrance and, thus, improve enzyme activity, which has been confirmed in this study (Figure 1D). During the molecular dynamic simulations, the RMSD of each complex with the reference of the docking conformation was also calculated. The RMSDs of PhoA, DelSigphoA, and DelSigD ${ }_{153} \mathrm{G}_{-} \mathrm{D}_{330} \mathrm{~N}$ were maintained at 3.04.5, 2.5-3.0, and 3.0 $\AA$, respectively. The results showed that the mutations had no effect on the structural stability. Therefore, sitedirected mutagenesis increases the activity of the enzyme without affecting the structural stability.

It was also observed that these plasmids were unstable and rapidly lost from the cells, which were growing without antibiotics. In this study, the toxin-antitoxin system (mazEmazF system) (Zhang et al., 2006: e71) was introduced into the expression plasmid by Red/ET recombineering. The MazE was the antidote to the toxin MazF of E. coli. Colony-forming unit counting was also performed to test the plasmid stability in the strains, and the results revealed that the stability of DelSigD ${ }_{153} \mathrm{G}-\mathrm{D}_{330} \mathrm{~N}$ mutants has been significantly improved (Supplementary Figure 2).

\section{Precipitation of Extracellular Proteins Including Alkaline Phosphatase}

To purify the AKP, we first detected the enzyme activity of the supernatant and the centrifugal pellets. The result revealed that the enzymatic activity of the supernatant accounted for the majority (Figure 2A). The AKP enzyme protein was precipitated by acid, acetone, ethanol, and salt precipitation. No enzyme activity was detected at $\mathrm{pH} 4,4.5,5,5.5,6$, or with salt precipitation treatment. Conversely, a certain activity was observed by the treatment with acetone or ethanol, and the highest precipitation was observed with acetone treatment. Therefore, it can be inferred that acetone was the best reagent for AKP enzyme precipitation (Figure 2B). The collection of high concentrations of AKP requires mass culture. The supernatant of DelSigpho $A$ and DelSigD ${ }_{153} \mathrm{G}_{-} \mathrm{D}_{330} \mathrm{~N}$ was treated with different proportions of acetone. We found that within a certain range, the greater the dosage of acetone, the AKP enzyme activity would be the higher (Figure 2C). The acetone treatment time was optimized to $50 \%(\mathrm{v} / \mathrm{v})$ of the acetone concentration. The results revealed that within a certain range, the longer the treatment 


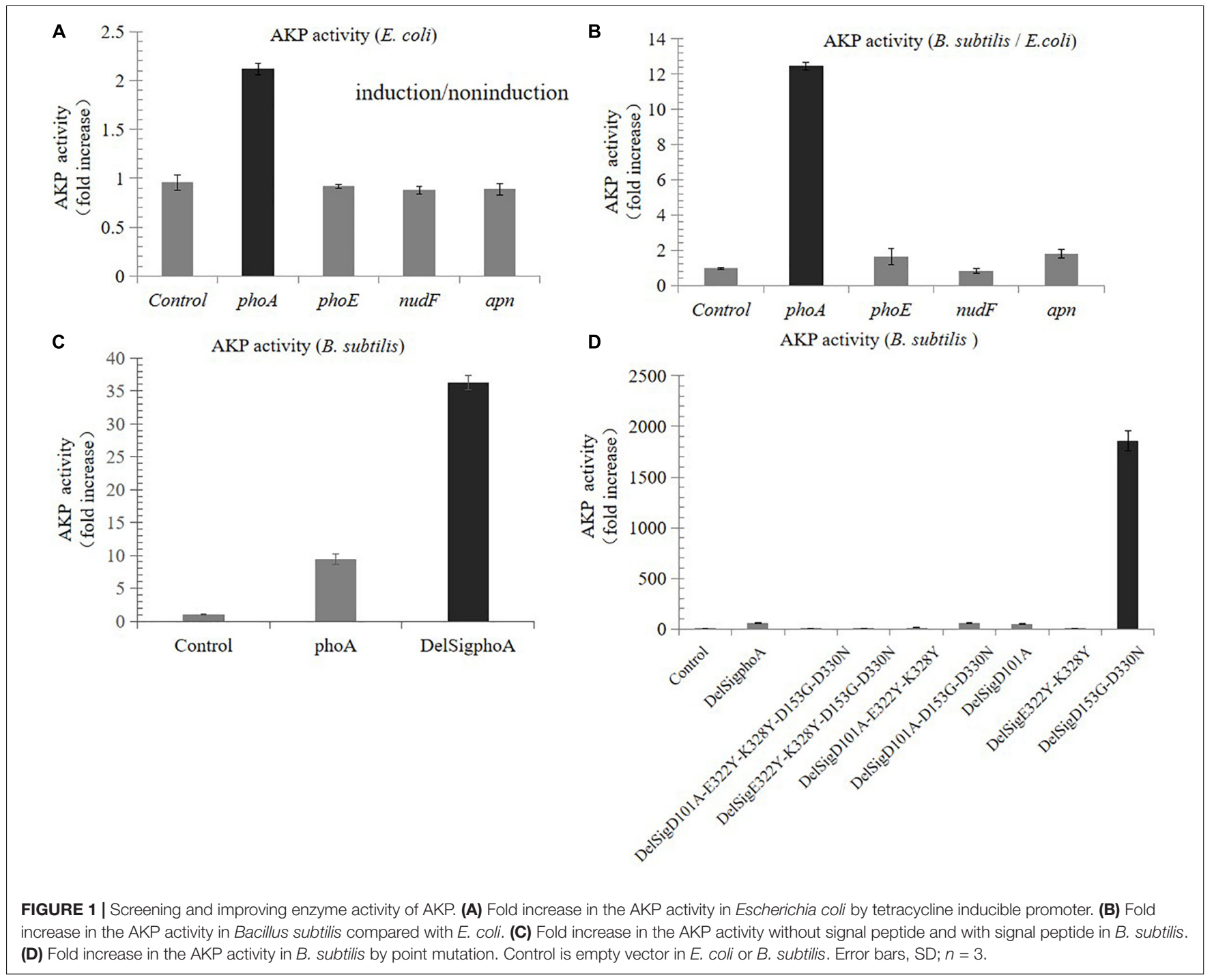

time, the higher the activity of AKP, and it reached the peak at 30 min (Figure 2D).

\section{Thermal Stability of Alkaline Phosphatase}

The thermal stability of AKP was also tested at high temperature. When the temperature was at $60^{\circ} \mathrm{C}$, the relative activity of DelSigphoA was significantly reduced and only retaining $40 \%$ of its activity. The enzyme activity of the DelSigD ${ }_{153} \mathrm{G}-\mathrm{D}_{330} \mathrm{~N}$ did not change significantly. The enzyme activity of both constructs still retained $20 \%$ with increasing temperature up to $120^{\circ} \mathrm{C}$ (Figure 3A). Normal spray drying was retained for $5 \mathrm{~s}$ at high temperature. The activity of the construct was detected by shortening the treatment time at $120^{\circ} \mathrm{C}$. After $1 \mathrm{~min}$ of treatment, the enzyme activity of DelSigphoA decreased by $60 \%$, while that of DelsigD ${ }_{153} \mathrm{G}_{-} \mathrm{D}_{330} \mathrm{~N}$ decreased by only $20 \%$. The results showed that the thermal stability of DelSigD ${ }_{153} \mathrm{G}-\mathrm{D}_{330} \mathrm{~N}$ was superior to that of DelSigphoA, and it still maintains higher enzymatic activity even after $1 \mathrm{~min}$ of treatment at $120^{\circ} \mathrm{C}$
(Figure 3B). As can be seen, the AKP appears to be dried at a relatively high temperature and retained high enzyme activity.

\section{Dynamic Simulation for Measuring the Alkaline Phosphatase Activity in vitro}

The AKP would pass through the digestive system of the piglet with different $\mathrm{pH}$ values (Figure $3 \mathrm{C}$ ); thus, an in vitro measurement system of the AKP activity was established. The AKP activity of DelSigphoA and DelSigD ${ }_{153} \mathrm{G}-\mathrm{D}_{330} \mathrm{~N}$ was tested in different $\mathrm{pH}$ ranges and revealed that no activity was detected for the DelSigphoA or DelSigD ${ }_{153} \mathrm{G}-\mathrm{D}_{330} \mathrm{~N}$ under acidic conditions (stomach: $\mathrm{pH}=2.3-2.5$ ). After adjusting the $\mathrm{pH}$ value simulated to intestinum tenue $(\mathrm{pH}=7-8)$, the AKP activity was restored to $20 \%$ and $30 \%$ for DelSigphoA and DelSigD ${ }_{153}$ G$\mathrm{D}_{330} \mathrm{~N}$, respectively. Subsequently, the $\mathrm{pH}$ value was changed into 6-7 simulated to intestinum crissum. The AKP activity was recovered to $55 \%$ and $65 \%$ for DelSigphoA and DelSigD ${ }_{153}$ G$\mathrm{D}_{330} \mathrm{~N}$, respectively (Figure 3D). The results suggested that the AKP was stable with a significant loss of enzyme activity under 

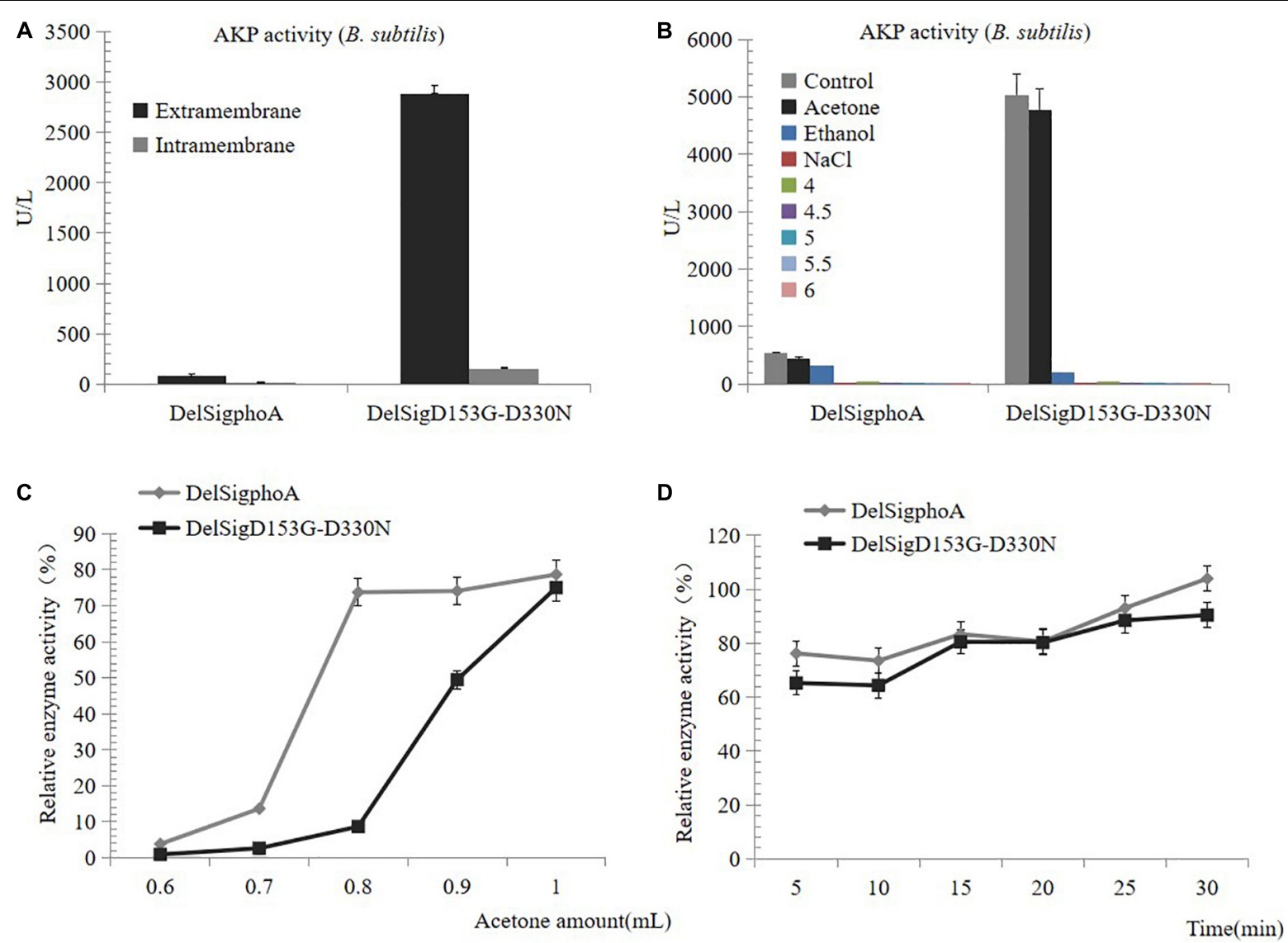

FIGURE 2 | Precipitation of AKP. (A) Distribution of AKP in fermentation broth. (B) Precipitation of AKP with acid, acetone, ethanol, and salt precipitation. (C) The effect of amount of acetone on AKP activity. (D) The effect of incubation time on AKP activity under acetone concentration (V/V) of 50\%. Error bars, SD; $n=3$.

strong acidic conditions. Meanwhile, its activity was resumed to a certain extent from acidic to alkaline conditions.

\section{The Effect of Alkaline Phosphatase on IPEC-J2 Cells}

The effect of AKP on cytokine gene expression in IPEC-J2 cells is shown in Figures 4A-C. Compared with the control, there was significant effect on the relative gene expression of IL-6, IL-8, and TNF- $\alpha$ in the LPS treatment $(p<0.01)$. The DelSigphoA treatment has no significant effect on IL-6 and IL-8, and downregulates the mRNA abundance of TNF$\alpha(p<0.05)$. Nevertheless, the DelSigD ${ }_{153} \mathrm{G}-\mathrm{D}_{330} \mathrm{~N}$ treatment extremely significantly downregulated the gene expression of IL6 , IL-8, and TNF- $\alpha(p<0.01)$. Compared with the control + LPS, the DelSigphoA + LPS and DelSigD ${ }_{153}$ G-D ${ }_{330} \mathrm{~N}+$ LPS treatments were extraordinarily significantly downregulated on the mRNA abundance of IL-6, IL-8, and TNF- $\alpha(p<0.01)$.

The effect of AKP on transporter gene expression in IPECJ2 cells is shown in Figure 4D. Compared with the control, LPS treatment was significantly upregulated on the relative gene expression of ASCT-2 $(p<0.01)$. Nevertheless, the DelSigphoA and DelSigD ${ }_{153} \mathrm{G}-\mathrm{D}_{330} \mathrm{~N}$ treatments significantly downregulated the gene expression of ASCT-2 $(p<0.01)$. Compared with the control + LPS, the DelSigphoA + LPS and DelSigD ${ }_{153}$ G$\mathrm{D}_{330} \mathrm{~N}+$ LPS treatments were extraordinarily significantly downregulated on ASCT-2 $(p<0.01)$.

The effect of AKP on gene expression of tight junction proteins in IPEC-J2 cells (Figures 4E,F). Compared with the control, LPS significantly downregulated the expression of ZO1 and CLDN-3 $(p<0.01)$. Nevertheless, the DelSigphoA and DelSigD ${ }_{153} \mathrm{G}-\mathrm{D}_{330} \mathrm{~N}$ treatment groups significantly upregulated the expression of ZO-1 and CLDN-3 $(p<0.01)$. Compared with the control + LPS, the DelSigphoA + LPS and DelSigD ${ }_{153}$ G$\mathrm{D}_{330} \mathrm{~N}+$ LPS treatment groups significantly upregulated the gene expression of ZO-1 and CLDN-3 ( $p<0.01)$.

\section{DISCUSSION}

Weaned piglets are prone to bacterial flora imbalance, excessive growth of harmful bacteria, and cause piglet diarrhea for changes in food status, nutritional composition, and physiological status. The additives could promote intestinal development, enhance immunity, increase production performance, and reduce the incidence of diarrhea in weaned pigs. Reasonable use of exogenous additives has important production significance for preventing and treating diarrhea. 
A

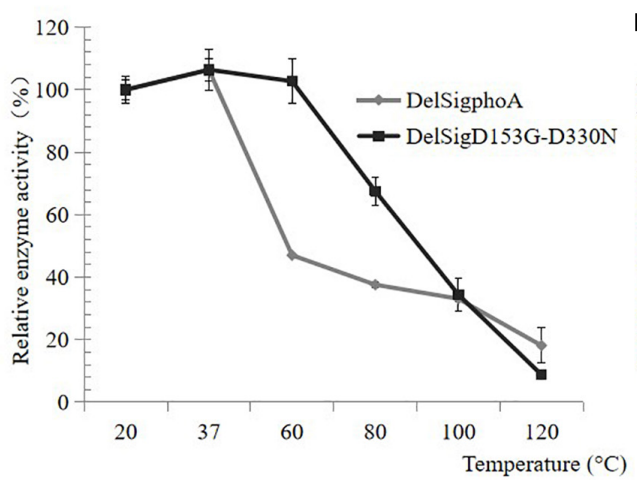

C

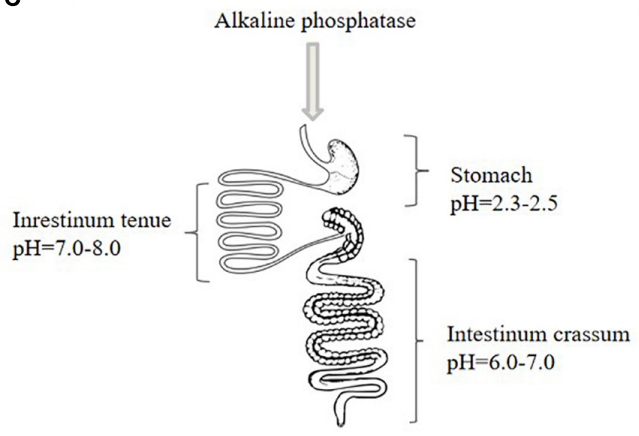

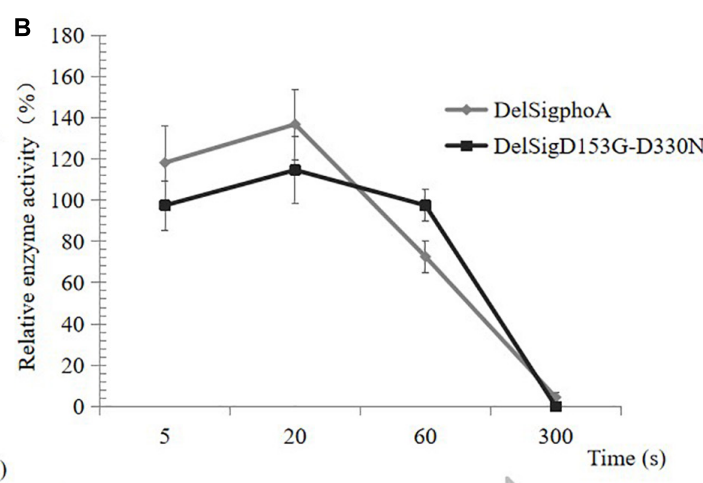

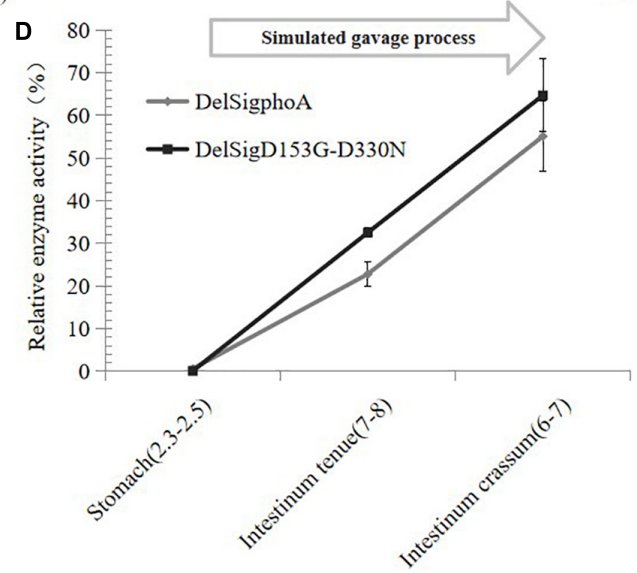

FIGURE 3 | Heat, acid, and alkali stability of AKP. (A) Heat stability of AKP on different temperatures. (B) The effect of incubation time at $120^{\circ} \mathrm{C}$ on AKP activity. Control is the vector. (C) Schematic diagram of piglet gastrointestinal after absorption of the AKP. (D) The effect of AKP on acid and alkali environment for simulating gavage process. Error bars, $\mathrm{SD} ; n=3$.

Previous studies have shown that AKP activity of the single mutants $\mathrm{D}_{330} \mathrm{~N}$ or $\mathrm{D}_{153} \mathrm{G}$ was three or five times higher than that of the wild-type phoA with the signal peptide in E. coli (Dealwis et al., 1995: 865-71, Le Du et al., 2002: 941-53). The activity of the double mutants $\mathrm{D}_{153} \mathrm{G}-\mathrm{D}_{330} \mathrm{~N}$ was 40 times higher than that of the wild-type $p h o A$ with the signal peptide in E. coli (Le Du et al., 2002: 941-53). Our study found that the activity of DelSigD ${ }_{153} \mathrm{G}-\mathrm{D}_{330} \mathrm{~N}$ in $B$. subtilis was up to 32 times more than the DelSigphoA, and nearly 1,600 times more than that of wild-type $p h o A$ with the signal peptide (Figure 1). In our study, the signal peptide was deleted, and B. subtilis was used as the expression host. Aggregation phenomenon and low expression level of the protein occur due to the presence of signal peptide, and transmembrane segments have been highlighted by a number of researchers (Masomian et al., 2017: 51-62). In addition, B. subtilis easily absorbs extracellular genetic material for horizontal gene transfer as a nutrient, and it also shows super-strong extracellular secretion ability of proteins and metabolites, and without producing endotoxins (Chen et al., 2005: 1456-60).

In order to enhance the stability of the enzyme and maintain its enzyme activity, various technologies have been used to form capsules including spray drying, spray cooling, extrusion coating, fluidized bed coating, liposome retention, coagulation, inclusion compounding, centrifugal extrusion, and rotating suspension separation (Gibbs et al., 1999: 213-24). In the spray drying of food, the hot air usually maintains $0.8 \sim 1.0 \mathrm{~s}$. In our study, the DelSigphoA and DelSigD ${ }_{153} \mathrm{G}_{-} \mathrm{D}_{330} \mathrm{~N}$ still maintained high activity after being treated at $120^{\circ} \mathrm{C}$ for $1 \mathrm{~min}$ in Figure 3B.

It is well known that the increase in pro-inflammatory factors leads to the destruction and localization of tight junction protein levels, increasing the passage of intestinal contents into the systemic circulation. Therefore, proinflammatory cytokine expression levels were widely considered to be an effective target for the treatment of inflammation (Liu et al., 2016: 1009-17). LPS triggers the inflammatory cascade by binding to TLR-4 on the apical membrane of PTEC, leading to the release in a variety of inflammatory mediators, including ATP and ADP, which further enhance the proinflammatory cascade through purine P2 receptor signaling (Peters et al., 2015: 4932-45). First of all, AKP dephosphorylates LPS for detoxification, thereby preventing TLR- 4 activation and reducing the production of proinflammatory factors (Liu et al., 2016: 1009-17). In addition, AKP dephosphorylates ATP and ADP to adenosine, thereby providing protection by reducing $\mathrm{P} 2$ receptor activation. This explains the reduction of proinflammatory cytokines when AKP alone was applied and the better performance of AKP in alleviating cellular inflammation under LPS stimulation, in 

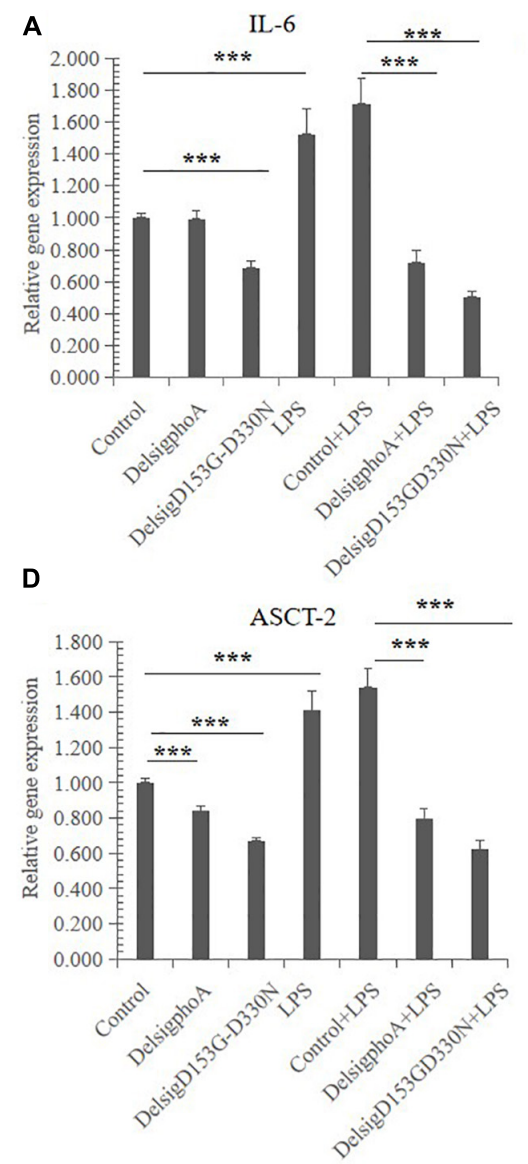

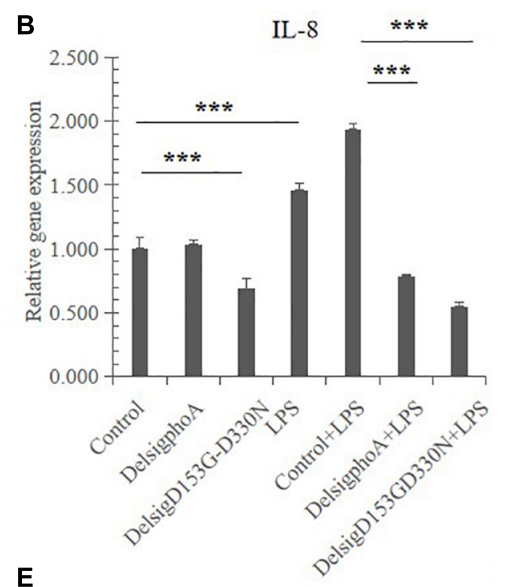

$\mathbf{E}$

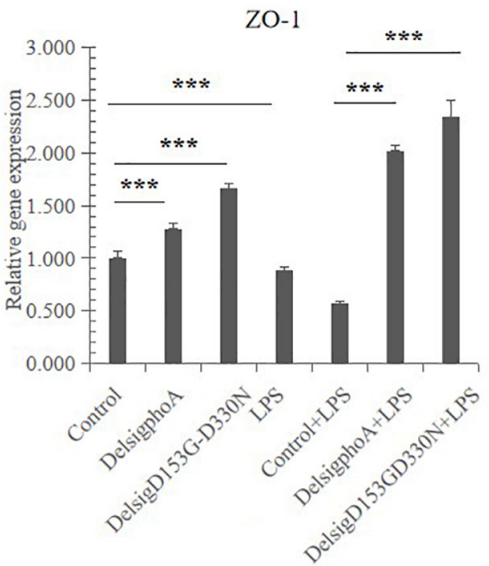

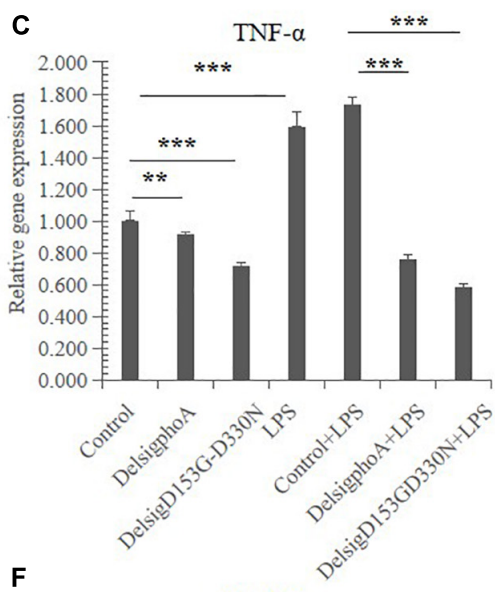

$\mathbf{F}$

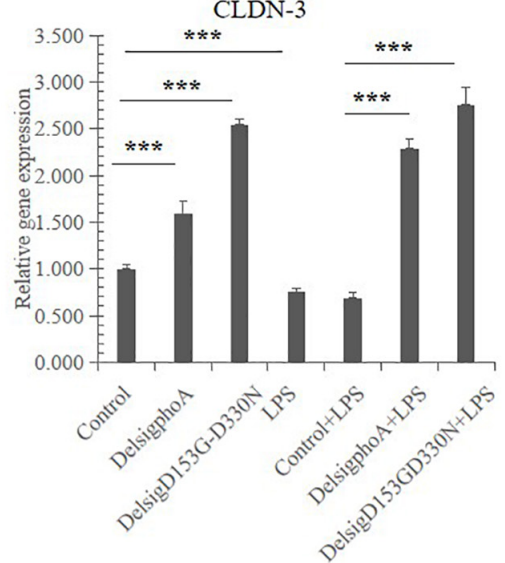

FIGURE 4 | The effect of AKP on relative mRNA abundances in LPS-induced IPEC-J2 cells. (A) The effect of AKP on relative mRNA abundances of interleukin 6 in LPS-induced IPEC-J2 cells. (B) The effect of AKP on relative mRNA abundances of interleukin 8 in LPS-induced IPEC-J2 cells. (C) The effect of AKP on relative mRNA abundances of tumor necrosis factor $\alpha$ in LPS-induced IPEC-J2 cells. (D) The effect of AKP on relative mRNA abundances of ASC amino acid transporter 2 in LPS-induced IPEC-J2 cells. (E) The effect of AKP on relative mRNA abundances of zonula occludens protein-1 in LPS-induced IPEC-J2 cells. (F) The effect of AKP on relative mRNA abundances of occludin-3 in LPS-induced IPEC-J2 cells. Error bars, SD; $n=10$. ${ }^{\star \star}$ Significant $(p<0.05)$, ${ }^{\star \star \star}$ very significant ( $\left.p<0.01\right)$.

correspondence with the previous study (Peters et al., 2015: 4932-45). Moreover, the adenosine activates AMPK through specific inhibitors to promote the maintenance of the integrity of the junction complex (Galardo et al., 2010: 49-58). However, there is no explanation, at present, why the tight junction protein expression is increased in cells treated with AKP and LPS than in cells treated with AKP alone. The adenosine is probably responsible for this phenomenon, but we need to further confirm it in the future. ASCT-2 is a key regulator of glutamine uptake in CD4 $+\mathrm{T}$ cells and influences the development of proinflammatory Th1 and Th17 responses in vivo and in vitro (Poffenberger and Jones, 2014: 635-7). AKP prevents the occurrence of proinflammatory reactions by downregulating the gene expression of ASCT2, consistent with the previous study (Xu et al., 2020: 111376). These results postulate that LPS exposure causes intestinal inflammation and apoptosis, thereby disrupting intestinal barrier function and affecting absorption and transport. AKP preconditioning may prevent LPS damage by regulating the mRNA abundance of transporter genes to some extent (Figure 4).

\section{DATA AVAILABILITY STATEMENT}

The original contributions presented in the study are included in the article/Supplementary Material, further inquiries can be directed to the corresponding author/s.

\section{AUTHOR CONTRIBUTIONS}

JiaY, HY, and JL contributed to the conceiving and designing of the experiments. YD and YX wrote the original draft of the manuscript. YD, JieY, YS, SY, QL, LW, DZ, YZ, CC, $\mathrm{QH}$, and YW performed the cell-treating trial and sample collection. YD conducted the strain screening and alkaline phosphatase assay. $\mathrm{YD}, \mathrm{YW}, \mathrm{CW}$, and $\mathrm{YZ}$ contributed to the protein structure simulation analysis experiment. JiaY, YX, MA, HY, and JL reviewed and revised the manuscript. JiaY obtained the funding and supervised the project. All authors have contributed to the article and approved the submitted version. 


\section{FUNDING}

This work was supported by the Scientific Research Fund of Hunan Provincial Education Department (21A0060), the Shandong Key Research and Development Program (2019JZZY010724), the Construction of Innovative Provinces in Hunan Province (2019RS3022), the National Students Platform for Innovation and Entrepreneurship Training Program (2020056), and the Shandong Key Research and Development Program (2019JZZY010724).

\section{ACKNOWLEDGMENTS}

We would like to thank Xin Yan from Nanjing Agricultural University for providing the pP43NMK plasmid.

\section{REFERENCES}

Bilski, J., Mazur-Bialy, A., Wojcik, D., Zahradnik-Bilska, J., Brzozowski, B., Magierowski, M., et al. (2017). The role of intestinal alkaline phosphatase in inflammatory disorders of gastrointestinal tract. Mediators Inflamm. 2017:9074601. doi: 10.1155/2017/9074601

Cai, D., Wang, H., He, P., Zhu, C., Wang, Q., Wei, X., et al. (2017). A novel strategy to improve protein secretion via overexpression of the SppA signal peptide peptidase in Bacillus licheniformis. Microb. Cell Fact. 16:70. doi: 10. 1186/s12934-017-0688-7

Case, D. A., Berryman, J. T., Betz, R. M., and Cerutti, D. S. (2015). Amber 2015, University of California, San Francisco, Vol. 3. San Francisco, CA: University of California, 1-884. doi: 10.13140/RG.2.2.32447.92320

Caspers, M., Brockmeier, U., Degering, C., Eggert, T., and Freudl, R. (2010). Improvement of Sec-dependent secretion of a heterologous model protein in Bacillus subtilis by saturation mutagenesis of the N-domain of the AmyE signal peptide. Appl. Microbiol. Biotechnol. 86, 1877-1885. doi: 10.1007/s00253-0092405-x

Chen, I., Christie, P. J., and Dubnau, D. (2005). The ins and outs of DNA transfer in bacteria. Science 310, 1456-1460. doi: 10.1126/science.1114021

Cui, W., Han, L., Suo, F., Liu, Z., Zhou, L., and Zhou, Z. (2018). Exploitation of Bacillus subtilis as a robust workhorse for production of heterologous proteins and beyond. World J. Microbiol. Biotechnol. 34:145. doi: 10.1007/s11274-0182531-7

Dealwis, C. G., Chen, L., Brennan, C., Mandecki, W., and Abad-Zapatero, C. (1995). 3-D structure of the D153G mutant of Escherichia coli alkaline phosphatase: an enzyme with weaker magnesium binding and increased catalytic activity. Protein Eng. 8, 865-871. doi: 10.1093/protein/8.9.865

Demain, A. L., and Vaishnav, P. (2009). Production of recombinant proteins by microbes and higher organisms. Biotechnol. Adv. 27, 297-306. doi: 10.1590/ S1415-47572004000300022

Estaki, M., DeCoffe, D., and Gibson, D. L. (2014). Interplay between intestinal alkaline phosphatase, diet, gut microbes and immunity. World J. Gastroenterol. 20, 15650-15656. doi: 10.3748/wjg.v20.i42.15650

Fairbrother, J. M., Nadeau, E., and Gyles, C. L. (2005). Escherichia coli in postweaning diarrhea in pigs: an update on bacterial types, pathogenesis, and prevention strategies. Anim. Health Res. Rev. 6, 17-39. doi: 10.1079/ahr2005105

Fu, J., Bian, X., Hu, S., Wang, H., Huang, F., Seibert, P. M., et al. (2012). Full-length RecE enhances linear-linear homologous recombination and facilitates direct cloning for bioprospecting. Nat. Biotechnol. 30, 440-446. doi: 10.1038/nbt.2183

Fu, L. L., Xu, Z. R., Shuai, J. B., Hu, C. X., Dai, W., and Li, W. F. (2008) High-level secretion of a chimeric thermostable lichenase from Bacillus subtilis by screening of site-mutated signal peptides with structural alterations. Curr. Microbiol. 56, 287-292. doi: 10.1007/s00284-007-9077-5

Gadde, U., Kim, W. H., Oh, S. T., and Lillehoj, H. S. (2017). Alternatives to antibiotics for maximizing growth performance and feed efficiency in poultry: a review. Anim. Health Res. Rev. 18, 26-45. doi: 10.1017/S1466252316000207

\section{SUPPLEMENTARY MATERIAL}

The Supplementary Material for this article can be found online at: https://www.frontiersin.org/articles/10.3389/fmicb. 2021.761189/full\#supplementary-material

Supplementary Figure 1 | The three initial structures ligand interaction diagram. Panel $\mathbf{( A )}$ is the binding pocket of AKP-ligand complex; panel $\mathbf{( B )}$ is the binding pocket of DelSigphoA-ligand complex; panel $\mathbf{( C )}$ is the binding pocket of DelSigD ${ }_{153} \mathrm{G}-\mathrm{D}_{330} \mathrm{~N}$-ligand complex. The yellow dash lines represent the hydrogen bonds, magenta dash lines represent salt bridges, cyan dash lines represent the $\mathrm{Pi}$ - Pi stacking interactions, green dash lines represent Pi-cation interactions.

Supplementary Figure 2 | Plasmid stability of $D_{153} G-D_{330} N$ in $B$. subtilis. The culture was transferred into the new LB medium, and the number of cells was counted on plates with and without kanamycin (resistance colony) in each generation after culturing for $12 \mathrm{~h}$. The ratio of kanamycin resistant clones to total cells was calculated. Error bars, SD; $n=3$.

Galardo, M. N., Riera, M. F., Pellizzari, E. H., Sobarzo, C., Scarcelli, R., Denduchis, B., et al. (2010). Adenosine regulates Sertoli cell function by activating AMPK. Mol. Cell. Endocrinol. 330, 49-58. doi: 10.1016/j.mce.2010.08.007

Gibbs, B. F., Kermasha, S., Alli, I., and Mulligan, C. N. (1999). Encapsulation in the food industry: a review. Int. J. Food Sci. Nutr. 50, 213-224. doi: 10.1080/ 096374899101256

Goldberg, R. F., Austen, W. G. Jr., Zhang, X., Munene, G., Mostafa, G., Biswas, S., et al. (2008). Intestinal alkaline phosphatase is a gut mucosal defense factor maintained by enteral nutrition. Proc. Natl. Acad. Sci. U.S.A. 105, 3551-3556. doi: $10.1073 /$ pnas.0712140105

Hou, G., and Cui, Q. (2012). QM/MM analysis suggests that Alkaline Phosphatase (AP) and nucleotide pyrophosphatase/phosphodiesterase slightly tighten the transition state for phosphate diester hydrolysis relative to solution: implication for catalytic promiscuity in the AP superfamily. J. Am. Chem. Soc. 134, 229-246. doi: $10.1021 /$ ja205226d

Jorgensen, W. L., Chandrasekhar, J., Madura, J. D., Impey, R. W., and Klein, M. L. (1983). Comparison of simple potential functions for simulating liquid water. J. Chem. Phys. 79, 926-935. doi: 10.1063/1.445869

Le Du, M. H., Lamoure, C., Muller, B. H., Bulgakov, O. V., Lajeunesse, E., Ménez, A., et al. (2002). Artificial evolution of an enzyme active site: structural studies of three highly active mutants of Escherichia coli alkaline phosphatase. J. Mol. Biol. 316, 941-953. doi: 10.1006/jmbi.2001.5384

Liu, L., Liu, Y., Shin, H. D., Chen, R. R., Wang, N. S., Li, J., et al. (2013). Developing Bacillus spp. as a cell factory for production of microbial enzymes and industrially important biochemicals in the context of systems and synthetic biology. Appl. Microbiol. Biotechnol. 97, 6113-6127. doi: 10.1007/s00253-0134960-4

Liu, W., Hu, D., Huo, H., Zhang, W., Adiliaghdam, F., Morrison, S., et al. (2016). Intestinal Alkaline Phosphatase regulates tight junction protein levels. J. Am. Coll. Surg. 222, 1009-1017. doi: 10.1016/j.jamcollsurg.2015.12.006

Masomian, M., Jasni, A. S., Rahman, R. N. Z. R. A., Salleh, A. B., and Basri, M. (2017). Impact of signal peptide and transmembrane segments on expression and biochemical properties of a lipase from Bacillus sphaericus 205y. J. Biotechnol. 264, 51-62. doi: 10.1016/j.jbiotec.2017.10.014

Molnár, K., Vannay, A., Szebeni, B., Bánki, N. F., Sziksz, E., Cseh, A., et al. (2012). Intestinal alkaline phosphatase in the colonic mucosa of children with inflammatory bowel disease. World J. Gastroenterol. 18, 3254-3259. doi: 10. 3748/wjg.v18.i25.3254

Morris, G. M., Huey, R., Lindstrom, W., Sanner, M. F., Belew, R. K., Goodsell, D. S., et al. (2009). AutoDock4 and AutoDockTools4: automated docking with selective receptor flexibility. J. Comput. Chem. 30, 2785-2791. doi: 10.1002/jcc. 21256

O'Neill, L. A., Golenbock, D., and Bowie, A. G. (2013). The history of Toll-like receptors - redefining innate immunity. Nat. Rev. Immunol. 13, 453-460. doi: 10.1038/nri3446

Omonijo, F. A., Liu, S., Hui, Q., Zhang, H., Lahaye, L., Bodin, J. C., et al. (2019). Thymol improves barrier function and attenuates inflammatory responses 
in porcine intestinal epithelial cells during Lipopolysaccharide (LPS)-induced inflammation. J. Agric. Food Chem. 67, 615-624. doi: 10.1021/acs.jafc.8b05480

Palócz, O., Pászti-Gere, E., Gálfi, P., and Farkas, O. (2016). Chlorogenic acid combined with Lactobacillus plantarum 2142 reduced LPS-induced intestinal inflammation and oxidative stress in IPEC-J2 Cells. PLoS One 11:e0166642. doi: 10.1371/journal.pone.0166642

Peters, E., Geraci, S., Heemskerk, S., Wilmer, M. J., Bilos, A., Kraenzlin, B., et al. (2015). Alkaline phosphatase protects against renal inflammation through dephosphorylation of lipopolysaccharide and adenosine triphosphate. $\mathrm{Br}$. J. Pharmacol. 172, 4932-4945. doi: 10.1111/bph.13261

Pié, S., Lallès, J. P., Blazy, F., Laffitte, J., Sève, B., and Oswald, I. P. (2004). Weaning is associated with an upregulation of expression of inflammatory cytokines in the intestine of piglets. J. Nutr. 134, 641-647. doi: 10.1093/jn/134.3.641

Poffenberger, M. C., and Jones, R. G. (2014). Amino acids fuel T cell-mediated inflammation. Immunity 40, 635-637. doi: 10.1016/j.immuni.2014.04.017

Ponder, J. W., and Case, D. A. (2003). Force fields for protein simulations. Adv. Protein Chem. 66, 27-85.

Schallmey, M., Singh, A., and Ward, O. P. (2004). Developments in the use of Bacillus species for industrial production. Can. J. Microbiol. 50, 1-17. doi: 10.1139/w03-076

Wojciechowski, C. L., Cardia, J. P., and Kantrowitz, E. R. (2002). Alkaline phosphatase from the hyperthermophilic bacterium T. maritima requires cobalt for activity. Protein Sci. 11, 903-911. doi: 10.1110/ps.4260102

Xu, X., Chang, J., Wang, P., Yin, Q., Liu, C., Li, M., et al. (2020). Effect of chlorogenic acid on alleviating inflammation and apoptosis of IPEC-J2 cells induced by deoxyniyalenol. Ecotoxicol. Environ. Saf. 205:111376. doi: 10.1016/j. ecoenv.2020.111376
Yang, J., and Zhang, Y. (2015). I-TASSER server: new development for protein structure and function predictions. Nucleic Acids Res. 43, W174-W181.

Yun, N., Kim, C., Cha, H., Park, W. J., Shibayama, H., Park, I. S., et al. (2013). Caspase-3-mediated cleavage of PICOT in apoptosis. Biochem. Biophys. Res. Commun. 432, 533-538. doi: 10.1016/j.bbrc.2013. 02.017

Zhang, X. Z., Yan, X., Cui, Z. L., Hong, Q., and Li, S. P. (2006). mazF, a novel counter-selectable marker for unmarked chromosomal manipulation in Bacillus subtilis. Nucleic Acids Res. 34:e71. doi: 10.1093/nar/gkl358

Conflict of Interest: The authors declare that the research was conducted in the absence of any commercial or financial relationships that could be construed as a potential conflict of interest.

Publisher's Note: All claims expressed in this article are solely those of the authors and do not necessarily represent those of their affiliated organizations, or those of the publisher, the editors and the reviewers. Any product that may be evaluated in this article, or claim that may be made by its manufacturer, is not guaranteed or endorsed by the publisher.

Copyright (c) 2022 Dong, Xia, Yin, Zhou, Sang, Yan, Liu, Li, Wang, Zhao, Chen, Huang, Wang, Abbasi, Yang, Wang, Li, Tu and Yin. This is an open-access article distributed under the terms of the Creative Commons Attribution License (CC BY). The use, distribution or reproduction in other forums is permitted, provided the original author(s) and the copyright owner(s) are credited and that the original publication in this journal is cited, in accordance with accepted academic practice. No use, distribution or reproduction is permitted which does not comply with these terms. 